\title{
RF and microwave channelizers based on microcombs
}

\author{
Xingyuan Xu, ${ }^{1}$ Mengxi Tan, ${ }^{1}$ Jiayang Wu, ${ }^{1}$ Andreas Boes, ${ }^{2}$ Thach G. Nguyen, ${ }^{2}$ Sai T. Chu, ${ }^{3}$ Brent E. Little, ${ }^{4}$ Roberto Morandotti, \\ Arnan Mitchell, ${ }^{2}$ and David J. Moss ${ }^{1, *}$ \\ ${ }^{I}$ Optical Sciences Centre, Swinburne University of Technology, Hawthorn, VIC 3122, Australia \\ ${ }^{2}$ School of Engineering, RMIT University, Melbourne, VIC 3000, Australia \\ ${ }^{3}$ Department of Physics and Material Science, City University of Hong Kong, Tat Chee Avenue, Hong Kong, China. \\ ${ }^{4} X i$ 'an Institute of Optics and Precision Mechanics Precision Mechanics of CAS, Xi' an, China. \\ ${ }^{5}$ INRS -Énergie, Matériaux et Télécommunications, 1650 Boulevard Lionel-Boulet, Varennes, Québec, J3X 1S2, Canada.
} *dmoss@swin.edu.au

Abstract - We demonstrate broadband RF channelizers based on microcombs with $49 \mathrm{GHz}$ and $200 \mathrm{GHz}$ FSRs. Up to 92 parallel channels and an instantaneous bandwidth of up to $8.08 \mathrm{GHz}$ are achieved, together with high-resolution RF spectral channelization and shaping. This approach is promising for high-performance integrated photonic $\mathrm{RF}$ receivers and processors.

Keywords-microwave photonic signal processing; Kerr frequency comb; microring resonator

\section{INTRODUCTION}

In modern photonic RF systems, to detect and analyze signals with powerful and flexible digital domain tools, the broadband signal needs to be spectrally sliced into digitalcompatible segments for separate digital processing. This is achieved by RF channelizers, and while electronic RF channelizers are subject to bandwidth limitations, photonic approaches offer ultra-large bandwidths, low transmission loss and strong immunity to electromagnetic interference [1].

Extensive effort has been made on photonic RF channelizers including those rely on a large number of spectrally dense and precisely centered narrow-linewidth filters [2], and others that employ multi-wavelength sources and optical periodic filters [3] Recently, microcombs [4] have attracted attention since they offer a large number of coherent wavelength channels in a $\mathrm{mm}^{2}$ size footprint. They have powered a wide range of RF applications [5] such as RF true time delays [6], transversal signal processors [7-9], frequency conversion [10], phaseencoded signal generators [11], and channelizers [12, 13].

Recently, [12] we reported an RF channelizer based on a $200 \mathrm{GHz}$ FSR microcomb combined with a $49 \mathrm{GHz}$ passive filter. This offered many attractive features such as high resolution, but was limited by the large comb spacing. Here, we report wideband RF channelizers using microcombs with a free spectral range (FSR) of $49 \mathrm{GHz}$ combined with a passive MRR filter with the same FSR that features a Q factor of $1.55 \times 10^{6}$. We realize RF channelizers with a large number of channels (92) and an RF instantaneous bandwidth $8.08 \mathrm{GHz}$. In addition, 117 MHz-resolution RF spectral shaping can also be achieved by combining all the channels together. We compare both approaches and show that this method offers a reduced footprint, lower complexity, and potentially lower cost.

\section{OPERATION PRINCIPLE}

Figure 1 shows the setup of our broadband RF channelizer that consists of three modules. The first module is microcomb generation and flattening, where an active MRR is pumped by a continuous-wave $(\mathrm{CW})$ laser to initiate parametric oscillation. With the MRR's high Q factor of over 1 million, the high nonlinear figure of merit, and tailored anomalous waveguide dispersion, sufficient parametric gain can be offered to generate Kerr frequency combs. The state of the generated frequency comb is determined mainly by the detuning between the pump and the resonance, and the pump power. As such, by sweeping the pump wavelength from blue to red, diverse nonlinear dynamic states, including the coherent soliton states, can be triggered. An optical spectral shaper (the commercially available Waveshaper) is then used to flatten the power of the comb lines to achieve equalized channel power.

In the second module, the flattened comb lines are directed to an electrooptical phase modulation, where the input broadband RF signal is multicast onto all the wavelength channels. Next, the replicated RF spectra are sliced by a passive

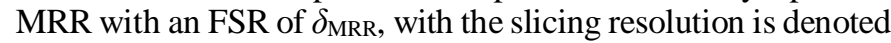
by the $3 \mathrm{~dB}$ bandwidth of the passive MRR. As a result, the RF spectral segments on all wavelength channels are effectively channelized with a progressive $\mathrm{RF}$ centre frequency, with $\left(\delta_{\mathrm{OFC}}\right.$ $-\delta_{\text {MRR }}$ ) corresponding to the channelized RF frequency step between adjacent wavelength channels. We note that by using phase modulation and notch filtering (i.e., the transmission of the passive MRR's through port) to achieve phase-modulation to intensity-modulation conversion, no other physical local oscillator paths would be required to achieve coherent homodyne detection. At last, the wavelength channels are demultiplexed and converted back into electrical domain separately for ADCs and further digital domain processing.

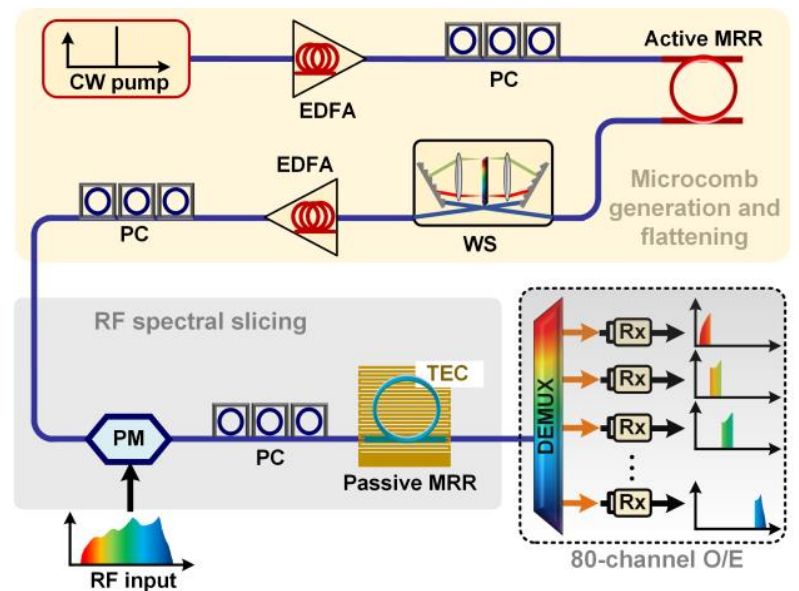


Fig. 1. Schematic diagram of the broadband RF channelizer based on a soliton crystal microcomb. EDFA: erbium-doped fibre amplifier. PC: polarization controller. MRR: micro-ring resonator. WS: Waveshaper. PM: phase modulator. TEC: temperature controller. DEMUX: de-multiplexer. Rx: wavelength channels, the corresponding power of each RF spectral segments can be arbitrarily controlled with a resolution given by the bandwidth of the passive MRR (117 MHz in [13]).
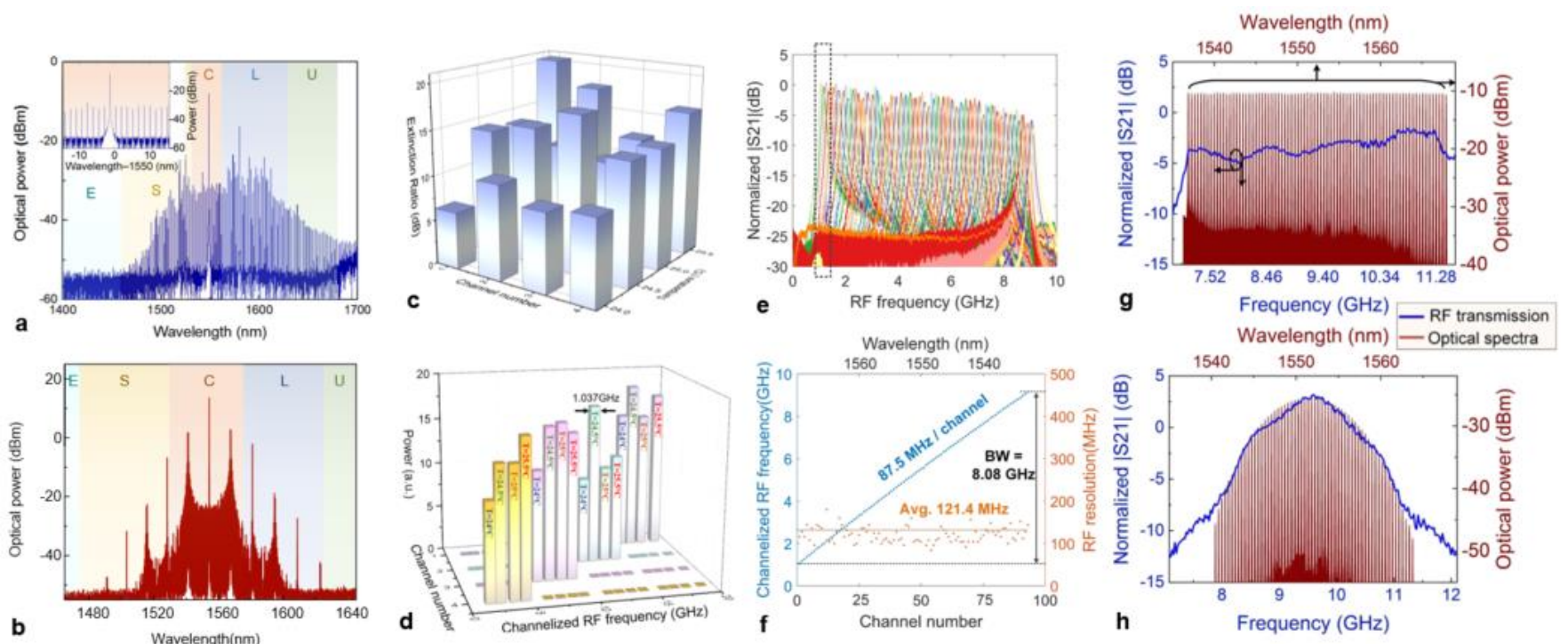

Fig. 2. Optical spectra of the microcombs with (a) $200 \mathrm{GHz}$ spacing and (b) $50 \mathrm{GHz}$ spacing. (c, d) Extracted channelized RF frequencies using the $200 \mathrm{GHz}$ microcomb. (e, f) Measured RF transmission spectra of the 92 channels using the $49 \mathrm{GHz}$ microcomb. (g, h) Demonstrated programmable RF spectral shaping using the bandwidth scaling technique.

Receiver

\section{RESULTS}

The active and passive MRRs were both fabricated in a CMOS-compatible doped silica glass platform [4]. During comb generation, the pump power was boosted with the wavelength swept manually from blue to red. As the detuning between the pump wavelength and the active MRR's resonance became small enough to ensure sufficient modulation-instability gain in the active MRR, single-FSR spacing microcombs can be generated. We note that a coherent soliton crystal state was generated by the $49 \mathrm{GHz}$ microcomb, enabled by the modecrossing induced background wave. Owing to the small FSR, the $49 \mathrm{GHz}$ microcomb offers up to 92 wavelength channels in the $\mathrm{C}$ band, in contrast to only 20 for the $200 \mathrm{GHz}$ microcomb.

Figure 2(c,d) shows the RF channelization results [12] for the $200 \mathrm{GHz}$ microcomb, with an RF range up to $19 \mathrm{GHz}$ assisted by thermal tuning, although the mismatch in the FSRs of the microcomb and the passive filtering MRR $(49 \mathrm{GHz})$ was too large to achieve continuous RF operational bands, resulting in only 4 channels being demonstrated [12]. Here, we employ the $49 \mathrm{GHz}$ soliton crystal microcomb as the multiwavelength source, in combination with another $49 \mathrm{GHz}$ MRR as the passive periodic filter. Due to the slightly different FSR mismatch between the two MRRs (87.5 MHz), continuous RF spectral channelization was achieved, with a resolution of $121.4 \mathrm{MHz}$ (determined by the $3 \mathrm{~dB}$ bandwidth of the passive MRR) and an instantaneous bandwidth of $8.08 \mathrm{GHz}$, which is effectively 22 times larger than the previous work [12] due to the larger number of channels (92). Next, we employed the $49 \mathrm{GHz}$ microcombbased channelizer to implement RF spectral shaping. By simply removing the demultiplexer and simultaneously summing all wavelength channels upon photodetection, RF bandwidth scaling can be achieved, thus by controlling the weights of the
Fig. 2(g, h) show the achieved RF transmission spectra and the corresponding optical spectra of the comb lines. Finally, components such as tunable dispersion compensators can provide flexible and compact and tunable alternatives to fibre spools for the dispersive delay elements[14-18].

In conclusion, we demonstrate broadband RF channelizers based on microcombs with $49 \mathrm{GHz}$ and $200 \mathrm{GHz}$ FSRs, achieving up to 92 parallel channels and an instantaneous bandwidth of up to $8.08 \mathrm{GHz}$. High-resolution RF spectral channelization and shaping were demonstrated, verifying our approach's feasibility and high performance.

\section{REFERENCES}

[1] J. Capmany and D. Novak, "Microwave photonics combines two worlds," Nat. Photonics, vol. 1, no. 6, pp. 319-330, Jun. 2007.

[2] S. T. Winnall et al., "A microwave channelizer and spectroscope based on an integrated optical Bragg-grating Fabry-Perot and integrated hybrid Fresnel lens system," IEEE T. Microw. Theory, vol. 54, no. 2, pp. 868872, Feb. 2006.

[3] X. Xie et al., "Broadband Photonic RF Channelization Based on Coherent Optical Frequency Combs and I/Q Demodulators," IEEE Photonics J., vol. 4, no. 4, pp. 1196-1202, Aug. 2012.

[4] A. Pasquazi et al., "Micro-Combs: A Novel Generation of Optical Sources", Physics Reports, vol. 729, pp. 1-81, Jan. 2018.

[5] X. Xu et al., "Microcomb-based photonic RF signal processing," IEEE Photonics Technology Letters, vol. 31, no. 23, pp. 1854-1857. 2019.

[6] X. Xu et al., "Photonic microwave true time delays for phased array antennas using a $49 \mathrm{GHz}$ FSR integrated optical micro-comb source," Photonics Research, vol. 6, no. 5, pp. B30-B36, May 1. 2018.

[7] X. Xu et al., "Advanced RF and microwave functions based on an integrated optical frequency comb source," Optics Express, vol. 26, no. 3, pp. 2569-2583, Feb 5. 2018.

[8] X. Xu et al., "Advanced Adaptive Photonic RF Filters with 80 Taps Based on an Integrated Optical Micro-Comb Source," Journal of Lightwave Technology, vol. 37, no. 4, pp. 1288-1295, Feb. 2019.

[9] M. Tan et al., "Microwave and RF Photonic Fractional Hilbert Transformer Based on a $50 \mathrm{GHz}$ Kerr Micro-Comb," Journal of Lightwave Technology, vol. 37, no. 24, pp. 6097-6104. 2019. 
[10] X. Xu et al., "Micro-comb based photonic local oscillator for broadband microwave frequency conversion," Journal of Lightwave Technology, vol. 38, no. 2, pp. 332-338. 2020.

[11] X. Xu et al., "Photonic RF phase-encoded signal generation with a microcomb source," Journal of Lightwave Technology, vol.38, no.7, pp.1722-1727. 2020. DOI: 10.1109/JLT.2019.2958564

[12] X. Xu et al., "Broadband RF Channelizer based on an Integrated Optical Frequency Kerr Comb Source," Journal of Lightwave Technology, vol. 36, no. 19, pp. 4519-4526. 2018.

[13] X. Xu et al., "High performance RF filters via bandwidth scaling with Kerr micro-combs," APL Photonics, vol. 4, no. 2, pp. 026102. 2019.

[14] D.J. Moss et al.,"Tunable dispersion and dispersion slope compensators for $10 \mathrm{~Gb} / \mathrm{s}$ using all-pass multicavity etalons", IEEE Photonics Technology Letters, vol. 15, no. (5), 730-732 (2003).

[15] D.J. Moss et al., "Multichannel tunable dispersion compensation using all-pass multicavity etalons", paper TuT2 Optical Fiber Communications Conference, Anaheim (2002). Post-conference Technical Digest (IEEE Cat. No.02CH37339). Opt Soc. America. Part vol.1, 2002, pp. 132-3. Washington, DC, USA.

[16] D.J. Moss et al.,"Tunable dispersion compensation at $10 \mathrm{~Gb} / \mathrm{s}$ and $40 \mathrm{~Gb} / \mathrm{s}$ using multicavity all-pass etalons", Optical Fiber Communications Conference (OFC) paper TuD1, page 162, Atlanta, GA, March (2003). Post-conference Digest (IEEE Cat. No.03CH37403). Opt. Soc. America. Part vol.1, 2003, pp. 162-3. Washington, DC, USA.

[17] L. Lunardi et al.,"Tunable dispersion compensators based on multi-cavity all-pass etalons for $40 \mathrm{~Gb} / \mathrm{s}$ systems", Journal of Lightwave Technology, vol. 20, no. 12, 2136 (2002). DOI: 10.1109/JLT.2002.806768.

[18] L.M. Lunardi et al., "An Etalon-Based Tunable Dispersion Compensator Device for 40-Gbit/s Applications", European Conf. on Optical Communications, Paper 5.4.6 Copenhagen, Sept. (2002). vol. 2, 2002, Piscataway, NJ, USA. Print ISBN: 87-90974-63-8. 